MATHEMATICS OF COMPUTATION

Volume 76, Number 257, January 2007, Pages 179-204

S 0025-5718(06)01896-5

Article electronically published on September 15, 2006

\title{
OPTIMAL ANISOTROPIC MESHES FOR MINIMIZING INTERPOLATION ERRORS IN $L^{p}$-NORM
}

\author{
LONG CHEN, PENGTAO SUN, AND JINCHAO XU
}

\begin{abstract}
In this paper, we present a new optimal interpolation error estimate in $L^{p}$ norm $(1 \leq p \leq \infty)$ for finite element simplicial meshes in any spatial dimension. A sufficient condition for a mesh to be nearly optimal is that it is quasi-uniform under a new metric defined by a modified Hessian matrix of the function to be interpolated. We also give new functionals for the global moving mesh method and obtain optimal monitor functions from the viewpoint of minimizing interpolation error in the $L^{p}$ norm. Some numerical examples are also given to support the theoretical estimates.
\end{abstract}

\section{INTRODUCTION}

In this paper, we are interested in obtaining (nearly) optimal meshes for the piecewise linear finite element interpolation of a given function. Let $\Omega \subset \mathbb{R}^{n}$ be a bounded domain, $\mathcal{T}_{N}$ a simplicial finite element mesh of $\bar{\Omega}$ with a fixed number $N$ of elements, and $u_{I}$ a piecewise linear finite element interpolation of a given function $u$ defined on $\bar{\Omega}$. We try to find an optimal mesh that minimizes the error $\left\|u-u_{I}\right\|_{L^{p}(\Omega)}(1 \leq p \leq \infty)$ in some sense.

This question can be traced back to de Boor [33, 34] where a problem of the best approximation by free knot splines was studied in one spatial dimension. In this work, the equidistribution principle was first introduced, and this concept has been widely used by other researchers studying grid adaptation. A pioneering work in adaptive finite element methods was done by Babuška and Rheinboldt [9] in which a finite element mesh was shown to be nearly optimal in the sense of minimizing the error in the $H^{1}$ norm if the local errors are approximately equal for all elements. Thus, to get an optimal mesh, elements where the error are large will be marked for refinement, while elements with a small error are left unchanged or coarsened.

Most of the adaptive finite element methods in the literature (see [10]) are concerned with meshes that are shape regular (which in two dimensions means that no element has very small angle) and this type of shape-regular finite element are appropriate for physical problems that are mostly isotropic. But for some anisotropic

Received by the editor October 13, 2003 and, in revised form, November 23, 2005

2000 Mathematics Subject Classification. Primary 41A25, 41A50,65M15,65M50, 65M60, $65 \mathrm{~N} 15,65 \mathrm{M} 30,65 \mathrm{M} 50$.

The authors were supported in part by NSF Grant \#DMS-0074299 and Center for Computational Mathematics and Applications, Penn State University.

The third author was also supported in part by NSF DMS-0209497 and NSF DMS-0215392 and the Changjiang Professorship through Peking University. 
problems (with, say, sharp boundary layers or internal layers), the shape of elements can be further optimized and an equidistribution of a scalar error density is not sufficient to ensure that a mesh is optimally efficient [31. In two dimensions the optimal triangulations for the discontinuous piecewise linear approximation in the sense of minimizing error in the $L^{2}$ norm was studied by Nadler 70 . The $L^{\infty}$ case was studied by D'Azevedo and Simpson 31, 32. An optimal mesh obtained in those works is that each triangle under the Hessian metric is equilateral and the error is equidistributed on each triangle. Recently the optimality of the $L_{\infty}$ error estimates was shown by Agouzal, Lipnikov and Vassilevski [3, 2, 62, 61, 63. Anisotropic mesh adaptation which aims to generate equilateral triangles under the metric induced by the Hessian matrix was developed in [80, 16, 43, 36] and successfully applied to the computational fluid dynamic problems in two spatial dimensions [43, 37.

Another global approach has been carried out in the literature in the study of the so-called moving mesh method $[39,83,20,74,20,46,23,49,66,81,15,65,38,35,60$, 59. In this type of study, a given finite element grid (often uniform to begin with) is moved while keeping the topological structure to better approximate a solution that has a certain anisotropic property. While most of this line of works in the literature are heuristic, there has been some work recently on the theoretical understanding of how a moving finite element method works [46, 49, 38, 65. In Huang [46] and Huang and Sun [49, by formulating the conditions in terms of the isotropy and the equidistribution, the authors obtained (formally) interpolation error estimates in $L^{2}$ and $H^{1}$ norms and further constructed corresponding functionals to be minimized for the global moving mesh methods.

There are other a priori and a posteriori interpolation error estimates for anisotropic finite elements [5, 6, 7, 4, 40, 41, 57, 58, 51, 52, 54, 55, 53, 56, 67, 71, 72. Apel [4] obtained some estimates under a condition on the coordinate orientation and on the maximal allowable mesh angle. Formaggia and Perotto 40, exploited the spectral properties of the affine map from the reference triangle to the general triangle to get anisotropic estimates for the $L^{2}$ and $H^{1}$ interpolation error on linear finite elements in two dimensions. Kunert [51] introduced the matching function to measure the alignment of an anisotropic function and an anisotropic mesh and presented error estimates using the matching function. Yet the overall optimal convergent rate in terms of the number of degrees of freedom is not easy to get from those approaches.

In this paper we first present a new local edge-based error estimate and global interpolation error bound in $L^{p}(1 \leq p \leq \infty)$ norm for any spatial dimension and then justify the optimality of this error bound. This estimate can be viewed as a modification and generalization of the previous work mentioned above. We require the mesh to be quasi-uniform under the new metric $(\operatorname{det} H)^{-1 /(2 p+n)} H$ where $H$ is a majorant of the Hessian matrix $\nabla^{2} u$ (see Section 2 for the details). Specifically the simplices are locally isotropic and their volumes are globally equidistributed under the new metric. Combining with another appropriate technical assumption, we obtain the error estimate

$$
\left\|u-u_{I}\right\|_{L^{p}(\Omega)} \leq C N^{-2 / n}\|\sqrt[n]{\operatorname{det} H}\|_{L^{\frac{p n}{2 p+n}}(\Omega)},
$$

where $N$ is the number of elements in the triangulation and the constant $C$ does not depend on $u$ and $N$. This estimate is optimal in the sense that it is a lower bound if $u$ is strictly convex or concave. 
We would like to point out that the estimate (1.1) has been formally obtained for the special case $p=\infty$ in D'Azevedo [31] and for the case $p=2$ in Huang and Sun [49. We will derive the more general estimate (1.1) rigorously using a different approach in this paper. More importantly, we will use this general estimate to derive several new results for the moving mesh method.

Based on this new error bound, we can construct a nonlinear functional whose minimizer aims to satisfy the equidistribution and isotropy requirements simultaneously in the moving mesh method. Let $\Omega_{c}$ be the computational domain with a quasi-uniform (under the standard Euclidean metric) triangulation $\mathcal{T}_{N, c}$. The mesh on $\Omega$ can be viewed as the image of a transformation $x=x(\xi): \Omega_{c} \rightarrow \Omega$. Then that the transformed mesh is quasi-uniform with respect to the metric $G(x)$ is more or less equivalent to that $x=x(\xi)$ is the global minimizer of the minimizing problem

$$
\min _{x} \int_{\Omega_{c}}\left[\sum_{i=1}^{n}\left(\nabla x_{i}\right)^{t} G(x) \nabla x_{i}\right]^{q}(\xi) d \xi, \quad q>n / 2 .
$$

It is often more convenient to study the moving meshes in terms of $\xi=\xi(x)$. In this case, we have the optimization problem

$$
\min _{\xi} \int_{\Omega}(\operatorname{det} G)^{1 / 2}\left[\sum_{i=1}^{n}\left(\nabla \xi_{i}\right)^{t} G^{-1} \nabla \xi_{i}\right]^{q}(x) d x, \quad q>n / 2 .
$$

The minimizer of either of these two functionals is expected to satisfy, to some degree, both equidistribution and isotropy conditions simultaneously. We note that the $q=1$ case corresponds to the harmonic mapping but we require $q>n / 2$ here. When $n \geq 3$, these minimization problems (which are more or less $p$-Laplacian with $p>n$ ) are significantly different from the harmonic mapping which has been most commonly used in the literature for the moving mesh method [39, 20, 48, 59. If we choose $G=(\operatorname{det} H)^{-1 /(2 p+n)} H$ in these functionals, we can get a nearly optimal mesh which minimizes the interpolation error $\left\|u-u_{I}\right\|_{L^{p}(\Omega)}$ by solving the above optimization problems.

The rest of the paper is organized as follows. In Section 2 we develop the interpolation error estimate and show that for strictly convex or concave functions, it is also a lower bound. In Section 3 we present two new functionals for a moving mesh method and give several practical remarks and comparisons with existing approaches. Section 4 contains numerical experiments, where we show the existence of such near optimal meshes. Some concluding remarks are made in the last section.

\section{Optimal ANisotropic Meshes}

In this section we will present an interpolation error bound by using the Taylor expansion and a scaling of a modified Hessian matrix. This bound is optimal in the sense that for strictly convex functions it is also a lower bound. For simplicity of exposition most theoretical results in this paper are presented under the regularity assumption that $u \in \mathcal{C}^{2}(\bar{\Omega})$.

2.1. A majorant of the Hessian matrix and Taylor expansion. Given a function $u \in \mathcal{C}^{2}(\bar{\Omega})$, where $\Omega$ is an open set of $\mathbb{R}^{n}$, a symmetric positive definite 
matrix function $H \in(C(\bar{\Omega}))^{n \times n}$ is called a majorant of the Hessian matrix for $u$ if it satisfies

$$
\left|\xi^{t}\left(\nabla^{2} u\right)(x) \xi\right| \leq c_{0} \xi^{t} H(x) \xi, \quad \xi \in \mathbb{R}^{n}, x \in \Omega
$$

for some positive constant $c_{0}$.

One example of $H$ can be constructed as follows. First, we diagonalize the Hessian: $\nabla^{2} u=Q^{t} \operatorname{diag}\left(\sigma_{i}\right) Q$ and then define

$$
H=Q^{t} \operatorname{diag}\left(\left|\sigma_{i}\right|\right) Q+\delta I, \quad \delta \geq 0 .
$$

It is easy to see that this matrix $H$ is a majorant of the Hessian matrix of $u$ and it satisfies (2.1) for any $\delta>0$ with $c_{0}=1$. When $\nabla^{2} u$ is singular, a positive parameter $\delta$ is critical to control the variation of $H$. A careful analysis in Huang 47. shows that $\delta$ can also be used to control the ratio of mesh points in the singular region and smooth region of the function.

Given a simplex $\tau$ with vertices $\left\{a_{k}\right\}_{k=1}^{n+1}$, we denote the edge vector $l_{i j}=a_{i}-a_{j}$. Let us now derive a linear interpolant error estimate in terms of the majorant of the Hessian matrix.

\section{Lemma 2.1.}

$$
\left|\left(u-u_{I}\right)(x)\right| \leq \frac{c_{0}}{2} \sum_{j, k=1}^{n+1} l_{j k}^{t} H_{k}(x) l_{j k} \quad \forall x \in \tau
$$

where

$$
H_{k}(x)=2 \int_{0}^{1} t H\left(a_{k}(t)\right), \quad a_{k}(t)=a_{k}+t\left(x-a_{k}\right) .
$$

Proof. Let $\left\{\lambda_{k}(x)\right\}$ be the barycentric coordinates of $\tau$. By Taylor expansion,

$$
u_{I}-u=\frac{1}{2} \sum_{k=1}^{n+1} \lambda_{k}(x)\left(x-a_{k}\right)^{t}\left[2 \int_{0}^{1} t \nabla^{2} u\left(a_{k}(t)\right)\right]\left(x-a_{k}\right) .
$$

Note that $x-a_{k}=\sum_{i} \lambda_{i} l_{i k}$. We have

$$
\left|\left(u_{I}-u\right)(x)\right| \leq \frac{c_{0}}{2} \sum_{i, j, k=1}^{n+1} \lambda_{i} \lambda_{j} \lambda_{k} l_{i k}^{t} H_{k} l_{j k} .
$$

By the Cauchy-Schwarz inequality,

$$
\begin{aligned}
\left|\left(u_{I}-u\right)(x)\right| \leq & \frac{c_{0}}{2} \sum_{i, j, k=1}^{n+1} \lambda_{i} \lambda_{j} \lambda_{k} \sqrt{l_{i k}^{t} H_{k} l_{i k}} \cdot \sqrt{l_{j k}^{t} H_{k} l_{j k}} \\
\leq & \frac{c_{0}}{2}\left(\sum_{i, j, k=1}^{n+1} \lambda_{i} \lambda_{j} \lambda_{k} l_{i k}^{t} H_{k} l_{i k}\right)^{1 / 2} \\
& \cdot\left(\sum_{i, j, k=1}^{n+1} \lambda_{i} \lambda_{j} \lambda_{k} l_{j k}^{t} H_{k} l_{j k}\right)^{1 / 2} \\
= & \frac{c_{0}}{2} \sum_{j, k=1}^{n+1} l_{j k}^{t} H_{k} l_{j k} .
\end{aligned}
$$

In the third step, we use the fact $\sum_{i=1}^{n+1} \lambda_{i}=1$. 
2.2. Main theorem. Let $u \in \mathcal{C}^{2}(\bar{\Omega})$ and $H$ be a majorant of the Hessian of $u$ satisfying (2.1). Associated with $u$, we consider a triangulation $\mathcal{T}_{N}$ of $\Omega$ that is adaptively obtained according to the properties of $u$. Here the subscript $N$ indicates that the triangulation has $N$ elements. We will now make two major assumptions on $\mathcal{T}_{N}$ in its relation with $u$.

Our first assumption on $\mathcal{T}_{N}$ is a local one. We assume that the matrix $H$ does not change very much on each element.

(A1). There exist two positive constants $\alpha_{0}$ and $\alpha_{1}$ such that

$$
\alpha_{0} \xi^{t} H_{\tau} \xi \leq \xi^{t} H(x) \xi \leq \alpha_{1} \xi^{t} H_{\tau} \xi, \quad \xi \in \mathbb{R}^{n},
$$

where $H_{\tau}$ is the average of $H$ over $\tau$, namely

$$
H_{\tau}=\frac{1}{|\tau|} \int_{\tau} H(x) d x
$$

The above assumption is hard to satisfy where $\nabla^{2} u$ is nearly singular. In this event, the introduction of the relaxation parameter $\delta$ in (2.2) becomes critical for this assumption.

Given $1 \leq p \leq \infty$, we introduce a scaled majorant of the Hessian matrix as follows

$$
H_{p}=\mu_{p} H, \quad \mu_{p}=(\operatorname{det} H)^{-\frac{1}{2 p+n}} .
$$

Note that $H_{p}$ also defines a Riemannian metric on $\Omega$. We have a new edge length and element volume introduced by this metric. In particular, we denote by $|\Omega|_{H_{p}}$ the volume of $\Omega$ under this new metric. By definition,

$$
|\Omega|_{H_{p}}=\int_{\Omega}\left(\operatorname{det} H_{p}\right)^{1 / 2}(x) d x=\int_{\Omega}(\operatorname{det} H)^{\frac{p}{2 p+n}}(x) d x .
$$

Given a triangulation $\mathcal{T}_{N}$, we then define a discretization of $H_{p}$ by

$$
H_{\tau, p}=\mu_{\tau, p} H_{\tau}, \quad \mu_{\tau, p}=\left[\operatorname{det}\left(H_{\tau}\right)\right]^{-\frac{1}{2 p+n}}, \quad \forall \tau \in \mathcal{T}_{N} .
$$

The edge length under this new metric is defined by

$$
d_{\tau, i j}=\left(l_{i j}^{t} H_{\tau, p} l_{i j}\right)^{1 / 2} .
$$

It is more convenient to order them with one single index, say lexigraphically, i.e., $d_{k}, k=1,2, \ldots, n(n+1) / 2$.

We will denote

$$
|\tau|_{H_{\tau, p}}=\left(\operatorname{det} H_{\tau, p}\right)^{1 / 2}|\tau|, \quad \text { and } \quad|\Omega|_{H_{\tau, p}}=\sum_{\tau \in \mathcal{T}_{N}}|\tau|_{H_{\tau, p}} .
$$

Under the assumption (A1), it is clear that $|\Omega|_{H_{\tau, p}} \leq C\left(\alpha_{1}\right)|\Omega|_{H_{p}}$.

Our second assumption is both local and global. Let

$$
\operatorname{diam}_{H_{\tau, p}}(\tau)=\max _{x, y \in \tau}(x-y)^{t} H_{\tau, p}(x-y)
$$

be the diameter of $\tau$ under the new metric $H_{\tau, p}$. The following assumption means that $\mathcal{T}_{N}$ is quasi-uniform under the new metric induced by $H_{\tau, p}$. 
(A2). There exist two positive constants $\beta_{0}$ and $\beta_{1}$ such that

$$
\frac{\operatorname{diam}_{H_{\tau, p}}(\tau)}{|\tau|_{H_{\tau, p}}^{1 / n}} \leq \beta_{0} \quad \forall \tau \in \mathcal{T}_{N}
$$

and

$$
\frac{\max _{\tau \in \mathcal{T}_{N}}|\tau|_{H_{\tau, p}}}{\min _{\tau \in \mathcal{T}_{N}}|\tau|_{H_{\tau, p}}} \leq \beta_{1} .
$$

The inequality (2.9) means that each $\tau$ is shape-regular under the metric $H_{\tau, p}$, namely all edges of $\tau$ are of comparable size. This is related to the so-called isotropy criterion considered in [46]. The inequality (2.10) means that all elements $\tau$ are of comparable size (under the new metric). This is related to the so-called equidistribution criterion, considered in 31] and [46.

It is easy to see that (A2) implies that

$$
\sum_{i=1}^{n(n+1) / 2} d_{\tau, i}^{2} \leq \frac{n(n+1)}{2} \beta_{0}|\tau|_{H_{\tau, p}}^{2 / n} \quad \forall \tau \in \mathcal{T}_{N}
$$

and

$$
|\tau|_{H_{\tau, p}} \leq \beta_{1} N^{-1}|\Omega|_{H_{\tau, p}} \quad \forall \tau \in \mathcal{T}_{N} .
$$

In the statement of our main theorem below, we need to use

$$
\|u\|_{L^{r}(\Omega)}:=\left\{\int_{\Omega}|u|^{r} d x\right\}^{\frac{1}{r}}
$$

We note that $\|\cdot\|_{L^{r}}$ is not a norm when $0<r<1$.

Theorem 2.2. Let $u \in \mathcal{C}^{2}(\bar{\Omega})$ and the triangulation $\mathcal{T}_{N}$ satisfy assumptions (A1) and (A2). The error estimate

$$
\left\|u-u_{I}\right\|_{L^{p}(\Omega)} \leq C N^{-2 / n}\|\sqrt[n]{\operatorname{det} H}\|_{L^{\frac{p n}{2 p+n}}(\Omega)}, 1 \leq p \leq \infty,
$$

holds for some constant $C=C\left(n, p, c_{0}, \alpha_{0}, \alpha_{1}, \beta_{0}, \beta_{1}\right)$.

Proof. We first prove (2.11) for $p=\infty$. Let us assume $u-u_{I}$ attains the maximum at point $x^{*}$ and let $\tau^{*}$ be a simplex containing $x^{*}$. By Lemma 2.1 and assumptions (A1) and (A2), we have

$$
\left|\left(u-u_{I}\right)\left(x^{*}\right)\right| \leq C \sum_{i=1}^{n(n+1) / 2} d_{\tau^{*}, i}^{2} \leq C\left|\tau^{*}\right|_{H_{\tau}}^{2 / n} \leq C N^{-2 / n}|\Omega|_{H_{\tau}}^{2 / n} \leq C N^{-2 / n}|\Omega|_{H}^{2 / n} .
$$

The desired result then follows.

For $1 \leq p<\infty$,

$$
\left|\left(u-u_{I}\right)(x)\right| \leq C \sum_{j, k=1, j<k}^{n+1} l_{j k}^{t} H_{\tau} l_{j k}=C \mu_{\tau, p}^{-1} \sum_{i=1}^{n(n+1) / 2} d_{\tau, i}^{2} \leq C \mu_{\tau, p}^{-1}|\tau|_{H_{\tau, p}}^{2 / n} .
$$

Thus

$$
\int_{\tau}\left|\left(u-u_{I}\right)(x)\right|^{p} d x \leq C \mu_{\tau, p}^{-p}|\tau||\tau|_{H_{\tau, p}}^{2 p / n} .
$$

Note that

$$
|\tau|=\left(\operatorname{det} H_{\tau, p}\right)^{-1 / 2}|\tau|_{H_{\tau, p}}=\mu_{\tau, p}^{-n / 2}\left(\operatorname{det} H_{\tau}\right)^{-1 / 2}|\tau|_{H_{\tau, p}},
$$


thus

$$
\int_{\tau}\left|\left(u-u_{I}\right)(x)\right|^{p} d x \leq C \mu_{\tau, p}^{-(p+n / 2)}\left(\operatorname{det} H_{\tau}\right)^{-1 / 2}|\tau|_{H_{\tau, p}}^{\frac{2 p+n}{n}}=C|\tau|_{H_{\tau, p}}^{\frac{2 p+n}{n}},
$$

since by definition (2.6) of $\mu_{\tau, p}$,

$$
\mu_{\tau, p}^{-(p+n / 2)}\left(\operatorname{det} H_{\tau}\right)^{-1 / 2}=1 .
$$

By assumptions (A1) and (A2)

$$
\begin{aligned}
\int_{\Omega}\left|\left(u-u_{I}\right)(x)\right|^{p} d x & =\sum_{\tau \in T^{N}} \int_{\tau}\left|\left(u-u_{I}\right)(x)\right|^{p} d x \leq C \sum_{\tau \in T^{N}}|\tau|_{H_{\tau, p}}^{\frac{2 p+n}{n}} \\
& \leq C N^{-\frac{2 p}{n}}|\Omega|_{H_{\tau, p}^{n}}^{\frac{2 p+n}{n}} \leq C N^{-\frac{2 p}{n}}|\Omega|_{H_{p}^{\frac{2 p+n}{n}}}
\end{aligned}
$$

which yields the desired result.

Remark 2.3. We would like to point out that the above theorem can be improved and generalized in many ways. For example, for $p \neq \infty$, the assumption (A2) can be slightly relaxed. More precisely, the assumption (A2) can be replaced by the following.

(A2 $\left.{ }^{\prime}\right)$ There exists two piecewise constant functions $\beta_{0}$ and $\beta_{1}$ such that

$$
\begin{gathered}
\sum_{i=1}^{n(n+1) / 2} d_{\tau, i}^{2} \leq \beta_{0}(\tau)|\tau|_{H_{\tau, p}}^{2 / n} \quad \forall \tau \in \mathcal{T}_{N}, \\
|\tau|_{H_{\tau, p}} \leq \beta_{1}(\tau) N^{-1}|\Omega|_{H_{\tau, p}} \quad \forall \tau \in \mathcal{T}_{N},
\end{gathered}
$$

and

$$
\sum_{\tau} \beta_{0}(\tau) \beta_{1}^{\frac{2 p+n}{n}}(\tau) \leq \beta_{2}
$$

where $\beta_{2}$ is constant. Such a relaxation is of practical significance. This means that optimal error estimates are still valid on a mesh which has a few exceptional elements that do not satisfy the isotropic or equidistribution assumptions.

Remark 2.4. Suboptimal metric. In some literature (see [70, 43, 36]) only the metric induced by a majorant of the Hessian matrix (without using the scaling $\mu_{\tau, p}$ or using $\mu_{\tau, \infty}=1$ ) is used to get an anisotropic mesh optimization methodology. To see why it would work to some degree, let us assume the triangulation is quasi-uniform under the metric $H_{\tau}$. Then

$$
\begin{aligned}
\left\|u-u_{I}\right\|_{L^{p}(\Omega)}^{p} & \leq C \sum_{\tau}|\tau||\tau|_{H_{\tau}}^{2 p / n} \\
& \leq C N^{-2 p / n}|\Omega||\Omega|_{H_{\tau}}^{2 p / n} \\
& =C N^{-2 p / n}\left(\int_{\Omega} 1 d x\right)\left(\int_{\Omega}(\operatorname{det} H)^{1 / 2} d x\right)^{\frac{2 p}{n}} .
\end{aligned}
$$

This tells us that we can still get a reasonable convergent rate if we only use the majorant of the Hessian metric without scaling. But this is not optimal since, comparing Theorem 2.2,

$$
\left(\int_{\Omega} 1 d x\right)\left(\int_{\Omega}(\operatorname{det} H)^{1 / 2} d x\right)^{\frac{2 p}{n}} \geq\left(\int_{\Omega}(\operatorname{det} H)^{\frac{p}{2 p+n}} d x\right)^{\frac{2 p+n}{p n}} .
$$


On the other hand, from $|\tau||\tau|_{H_{\tau}}^{2 p / n}$ we can see that if $|\tau|_{H_{\tau}}$ are almost equal for every $\tau$, but $|\tau|$ are not supposed to be equal in general. Thus we cannot guarantee the global equidistribution condition by only using the Hessian metric. While by scaling the metric, we can achieve both local and global conditions simultaneously.

2.3. Optimality of the result: A lower bound. In this section, we shall show that the error estimate presented above is sharp in some sense for strictly convex (or concave) functions.

Let us first introduce some standard short-hand notation for multiple indices. A multi-index $\alpha$ is an $m$-tuple of nonnegative integers $\alpha=\left(\alpha_{1}, \alpha_{2}, \ldots, \alpha_{m}\right)$. The length of $\alpha$ is defined by $|\alpha|=\sum_{i=1}^{m} \alpha_{i}$. For a given vector $\left(x_{1}, x_{2}, \ldots, x_{m}\right)$, we define $x^{\alpha}=x_{1}^{\alpha_{1}} x_{2}^{\alpha_{2}} \cdots x_{m}^{\alpha_{m}}$.

Assume that we are given a strictly convex (or concave) function $u \in \mathcal{C}^{2}(\bar{\Omega})$, namely $\nabla^{2} u$ is positive (or negative) definite and continuous on $\bar{\Omega}$. We can define a metric $H=\nabla^{2} u$, its average $H_{\tau}$ and $H_{\tau, p}$ as before.

Then we define $d_{i j}^{2}=\left(a_{i}-a_{j}\right) H_{\tau}\left(a_{i}-a_{j}\right), i, j=1,2, \ldots, n+1$, and $d^{2}=$ $\left(d_{1}^{2}, d_{2}^{2}, \ldots, d_{m}^{2}\right)$, where $m=n(n+1) / 2$. Furthermore we will define

$$
\kappa_{0}(\tau)=\min _{\xi \in \mathbb{R}^{n}, x \in \tau} \frac{\xi^{t} H(x) \xi}{\xi^{t} H_{\tau} \xi}, \quad \text { and } \quad \kappa_{1}(\tau)=\min _{x \in \tau}\left[\frac{\operatorname{det} H_{\tau}}{(\operatorname{det} H)(x)}\right]^{1 / n} \text {. }
$$

Since we assume $u$ is strictly convex (or concave), the above functions are well defined.

For a given multi-index $\alpha=\left(\alpha_{1}, \alpha_{2}, \ldots, \alpha_{n(n+1) / 2}\right)$, we define a $(n+1) \times(n+1)$ symmetric matrix $B=\left(b_{i j}\right)$ by $b_{i i}=0, b_{i j}=\alpha_{(i-1) \times(n+1)+j}$, for $i<j$ and a new multi-index $\tilde{\alpha}=\left(\tilde{\alpha}_{1}, \tilde{\alpha}_{2}, \ldots, \tilde{\alpha}_{n+1}\right)$ by $\tilde{\alpha}_{i}=\sum_{j=1}^{n+1} b_{i j}$.

Now we are ready to present our lemmas.

Lemma 2.5. Let $u \in \mathcal{C}^{2}(\bar{\Omega})$ be a strictly convex function. We have

$$
\begin{aligned}
\left\|u-u_{I}\right\|_{L^{p}(\tau)}^{p} & \geq \kappa_{0}(\tau) \frac{p ! n !}{2^{p}(2 p+n) !}|\tau| \sum_{|\alpha|=p} \frac{\tilde{\alpha} !}{\alpha !} d_{\tau}^{2 \alpha}, \quad 1 \leq p<\infty, \\
\left\|u-u_{I}\right\|_{L^{\infty}} & \geq \frac{\kappa_{0}(\tau)}{2(n+1)^{2}} \sum_{i=1}^{m} d_{\tau, i}^{2}, \quad p=\infty .
\end{aligned}
$$

Proof. By (2.3) and (2.13)

$$
\begin{aligned}
\left|\left(u-u_{I}\right)(x)\right| & \geq \frac{\kappa_{0}(\tau)}{2} \sum_{j=1}^{n+1} \lambda_{j}(x)\left(x-a_{j}\right)^{t} H_{\tau}\left(x-a_{j}\right) \\
& =\frac{\kappa_{0}(\tau)}{2} \sum_{i, j, k=1}^{n+1}\left(\lambda_{i} \lambda_{j}\right)(x)\left(a_{i}-a_{j}\right) H_{\tau}\left(x-a_{j}\right) .
\end{aligned}
$$

Using the symmetry of the index $i$ and $j$, we can also write the inequality as

$$
\left|\left(u-u_{I}\right)(x)\right| \geq \frac{\kappa_{0}(\tau)}{2} \sum_{i, j, k=1}^{n+1}\left(\lambda_{i} \lambda_{j} \lambda_{k}\right)(x)\left(a_{j}-a_{i}\right) H_{\tau}\left(x-a_{i}\right) .
$$

Summing the above two inequalities gives us that

$$
\left|\left(u-u_{I}\right)(x)\right| \geq \frac{\kappa_{0}(\tau)}{2} \sum_{i, j=1, i<j}^{n+1}\left(\lambda_{i} \lambda_{j}\right)(x) d_{\tau, i j}^{2} .
$$


The inequality (2.14) can then be obtained by using the two elementary identities

$$
\left(\sum_{i=1}^{m} x_{i}\right)^{p}=\sum_{|\alpha|=p} \frac{p !}{\alpha !} x^{\alpha}
$$

and

$$
\int_{\tau} \lambda^{\alpha}(x) d x=\frac{\alpha ! n !}{(|\alpha|+n) !}|\tau|
$$

where $\lambda=\left(\lambda_{1}, \lambda_{2}, \ldots, \lambda_{n+1}\right)$.

For $p=\infty$, we choose $x_{\tau}$, the barycenter of $\tau$, in (2.16). Since $\lambda_{i}\left(x_{\tau}\right)=1 /(n+1)$ for $i=1, \ldots, m$, we get (2.15).

Lemma 2.6. Let

$$
E_{p}(x)=\sum_{\alpha,|\alpha|=p} \frac{\tilde{\alpha} !}{\alpha !} x^{\alpha}, \quad 1 \leq p<\infty .
$$

Then for any $x=\left(x_{1}, x_{2}, \ldots, x_{m}\right)$ with $x_{i}>0, i=1,2, \ldots, m$,

$$
E_{p}(x) \geq E_{p}\left(x^{*}\right)\left(\sum_{i=1}^{m} x_{i}\right)^{p} .
$$

When $P>1$ the equality holds if and only if $x=x^{*}$ with $x^{*}=(1 / m, 1 / m, \ldots, 1 / m)$.

Proof. When $p=1$ the assertion (2.17) is trivially true. We will prove the result for $p>1$. Let us first compute the minimum of $E_{p}(x)$ under the constraint $\sum_{i=1}^{m} x_{i}=$ 1. Let

$$
F(x, \lambda)=E_{p}(x)-\lambda\left(\sum_{i=1}^{m} x_{i}-1\right) .
$$

The critical point of $F$ satisfies

$$
\lambda=\partial_{1} E_{p}(x)=\partial_{2} E_{p}(x)=\cdots=\partial_{m} E_{p}(x) .
$$

Here for convenience in notation, we let $\partial_{i} E_{p}$ denote the partial derivative $\frac{\partial E_{p}}{\partial x_{i}}$. Since $E_{p}$ is symmetric with respect to $x_{i}$, namely

$$
E_{p}(\sigma(x))=E_{p}\left(\sigma^{2}(x)\right)=\cdots=E_{p}\left(\sigma^{m}(x)\right),
$$

where $\sigma$ is the cyclic permutation $\sigma\left(\left(x_{1}, x_{2}, \ldots, x_{m}\right)\right)=\left(x_{2}, \ldots, x_{m}, x_{1}\right)$, we then have

$$
\partial_{1} E_{p}(\sigma(x))=\partial_{2} E_{p}\left(\sigma^{2}(x)\right)=\cdots=\partial_{m} E_{p}\left(\sigma^{m}(x)\right)
$$

and conclude that the point $\left(x^{*}, \partial_{1} E_{p}\left(x^{*}\right)\right)$ is a critical point of $F$.

Note that $E_{p}(x)$ is $p$ th homogeneous. By differentiating the Euler formula $x \cdot \nabla E_{p}(x)=p E_{p}(x)$, we get $x \cdot\left(\nabla^{2} E_{p}\right)=(p-1) \nabla E_{p}(x)$ and thus

$$
x \cdot \nabla^{2} E_{p}(x) x^{t}=(p-1) x \cdot \nabla E_{p}=(p-1) p E_{p}(x)>0 .
$$

Since $F$ has the same quadratic part as $E_{p}$ as a function of $x$, we conclude that $F$ achieves the minimum at $x^{*}$ under the constraint $\sum_{i=1}^{m} x_{i}=1$.

By combining this result with the obvious identity

$$
E_{p}\left(\frac{x}{\sum_{i=1}^{m} x_{i}}\right)=\frac{E_{p}(x)}{\left(\sum_{i=1}^{m} x_{i}\right)^{p}},
$$

we complete the proof. 
The following lemma is a well-known geometry inequality between the total edge length and the volume of a simplex; for an example, see 69] (p.517).

\section{Lemma 2.7.}

$$
\sum_{i=1}^{m} d_{\tau, i}^{2} \geq \frac{n(n+1) n !^{2 / n}}{(n+1)^{1 / n}}|\tau|_{H_{\tau, p}}^{2 / n} .
$$

The equality holds if and only if $\tau$ is equilateral under the metric $H_{\tau, p}$.

For a family of triangulations $\left\{\mathcal{T}_{N}, N \geq N_{0} \in \mathbb{N}\right\}$ of $\Omega$ we define

$$
\begin{gathered}
h_{N}=\max _{\tau \in \mathcal{T}_{N}} \operatorname{diam}(\tau), \\
\kappa_{0}^{N}(x)=\kappa_{0}(\tau), \kappa_{1}^{N}(x)=\kappa_{1}(\tau), \quad x \in \tau
\end{gathered}
$$

and

$$
\rho_{N}=\frac{\max _{\tau, i} d_{\tau, i}}{\min _{\tau, i} d_{\tau, i}} .
$$

We say all edges $\left\{d_{\tau, i}\right\}$ are asymptotically equal if $\lim _{N \rightarrow \infty} \rho_{N}=1$.

Theorem 2.8. Assume that $u \in \mathcal{C}^{2}(\bar{\Omega})$ is a strictly convex (or concave) function and $\left\{\mathcal{T}_{N}\right\}$ are a family of triangulations of $\Omega$ satisfying $\lim _{N \rightarrow \infty} h_{N}=0$. Then

$$
\liminf _{N \rightarrow \infty} N^{2 / n}\left\|u-u_{I}\right\|_{L^{p}(\Omega), N} \geq C_{n, p}\|\sqrt[n]{\operatorname{det} H}\|_{L^{\frac{p n}{2 p+n}}(\Omega)},
$$

where

$$
\begin{aligned}
C_{n, p} & =\left(\sum_{\alpha,|\alpha|=p} \frac{\tilde{\alpha} !}{\alpha !}\right) \frac{p ! n !}{(2 p+n) !} \frac{n !^{2 p / n}}{(n+1)^{p / n}}, \quad 1 \leq p<\infty, \\
C_{n, \infty} & =\frac{n}{2(n+1)} \frac{n !^{2 / n}}{(n+1)^{1 / n}} .
\end{aligned}
$$

Furthermore the equality holds if all edges in $\left\{\mathcal{T}_{N}\right\}$ are asymptotically equal under the new metric, namely $\lim _{N \rightarrow \infty} \rho_{N}=1$.

Proof. We first prove the case $1 \leq p<\infty$. By (2.14) in Lemmas 2.6 and 2.7

$$
|\tau| E_{p}\left(d_{\tau}^{2}\right)=\mu_{\tau, p}^{-p}|\tau| E_{p}\left(d_{\tau}^{2}\right) \geq C \mu_{\tau, p}^{-p}|\tau|\left(\sum_{i=1}^{m} d_{\tau, i}^{2}\right)^{p} \geq C \mu_{\tau, p}^{-p}|\tau||\tau|_{H_{\tau, p}}^{2 p / n},
$$

where $\mu_{\tau, p}$ and $|\tau|_{H_{\tau, p}}$ are defined by (2.6) and (2.8). The constant $C$ only depends on $p$ and $n$ but not $\tau$. The equality holds if and only if the simplex $\tau$ is regular under the new metric $H_{\tau, p}$; i.e., all edges of $\tau$ are equal under the new metric $H_{\tau, p}$.

By (2.12) and the definition of $\mu_{\tau, p}$, we know that

$$
\left.\mu_{\tau}^{-p}|\tau||| \tau\right|_{H_{\tau, p}} ^{2 p / n}=|\tau|_{H_{\tau, p}}^{\frac{2 p+n}{n}}
$$

Consequently,

$$
\begin{aligned}
\left\|u-u_{I}\right\|_{L^{p}(\Omega)}^{p} & \geq C \sum_{\tau \in \mathcal{T}_{N}} \kappa_{0}(\tau)|\tau|_{H_{\tau, p}^{\frac{2 p+n}{n}}} \\
& \geq C \kappa_{0}^{N}(x) \kappa_{1}^{N}(x) \sum_{\tau \in \mathcal{T}_{N}}\left(\int_{\tau}[\sqrt[n]{\operatorname{det} H}]^{\frac{n p}{2 p+n}}(x) d x\right)^{\frac{2 p+n}{n}} \\
& \geq C N^{-2 p / n} \kappa_{0}^{N}(x) \kappa_{1}^{N}(x)\left(\int_{\Omega}[\sqrt[n]{\operatorname{det} H}]^{\frac{n p}{2 p+n}}(x) d x\right)^{\frac{2 p+n}{n}} .
\end{aligned}
$$


The last inequality becomes an equality if and only if all the volumes of elements under the new metric are equal. Furthermore, if all edges are equal under the new metric, we can achieve all the equalities.

Since $\nabla^{2} u$ is continuous, $\kappa_{0}^{N}(x) \kappa_{1}^{N}(x)$ are uniformly bounded and it approaches 1 when $N$ goes to $\infty$. The desired inequality of the theorem for $1 \leq p<\infty$ then follows.

For $p=\infty$, we make use of (2.15) in Lemma 2.6 and follow the same procedure to get the desired inequality.

The constants $C_{n, p}$ in (2.18) are useful in other related problems. In [25] we show that $C_{n, \infty}$ is closely related to the sphere covering problem and $C_{n, 1}$ to optimal polytopes approximation of convex bodies.

Remark 2.9. The strict convexity assumption of the function is to ensure $\nabla^{2} u$ is a Riemannian metric so that we can apply the geometry inequalities locally. For $p=\infty$ the strict convexity assumption can be relaxed to be nonsingular; i.e., the indefinite metric is allowed in this case (see $[73,63$ ).

\section{New optimization PROBlems For the MOVing MESh Method}

In this section, we shall apply the error estimate obtained above to derive some new optimization problem that can be used in the moving mesh method. The basic idea in our argument below is not new (see, for example, Huang [46]), but the optimization problems we will obtain appear to be new and especially appear to be more appropriate than those in the literature. The main new feature of our new optimization problems is that they address the isotropic and equidistribution criteria simultaneously.

The moving mesh method can be described by two different sets of domains, $\Omega_{c}$ and $\Omega$, and corresponding grids, $\mathcal{T}_{N, c}$ and $\mathcal{T}_{N}$, which are linked by a transformation $x=x(\xi): \Omega_{c} \mapsto \Omega$. Here $\Omega_{c}$ is known as the computational domain with a quasiuniform triangulation $\mathcal{T}_{N, c}$ with respect to the standard Euclidean metric and $\Omega$ is the physical domain with an adapted mesh $\mathcal{T}_{N}$. The adapted grid $\mathcal{T}_{N}$ can be viewed as a result of moving the grid $\mathcal{T}_{N, c}$ through the transformation:

$$
x=x(\xi), x=\left(x_{1}, x_{2}, \ldots, x_{n}\right)^{t}, \xi=\left(\xi_{1}, \xi_{2}, \ldots, \xi_{n}\right)^{t} .
$$

Let us assume $x: \Omega_{c} \mapsto \Omega$ is a differentiable homeomorphism and denote the Jacobian of the transformation $x=x(\xi): \Omega_{c} \mapsto \Omega$ by

$$
J=\frac{\partial x}{\partial \xi}=\left(\left(\nabla_{\xi} x_{1}\right)^{t}, \ldots,\left(\nabla_{\xi} x_{n}\right)^{t}\right)=\left(\frac{\partial x_{i}}{\partial \xi_{j}}\right) .
$$

Let $G$ be a metric on $\Omega$. Then the transformation induces a new metric on $\Omega_{c}$ which is $G_{c}=J^{t} G J$. Now we have three manifolds $(\Omega, G),\left(\Omega_{c}, I\right)$, and $\left(\Omega_{c}, G_{c}\right)$, where $I$ denotes the standard Euclidean metric. $\mathcal{T}_{N}$ is quasi-uniform in $(\Omega, G)$ if $\mathcal{T}_{N, c}$ is quasi-uniform in $\left(\Omega_{c}, G_{c}\right)$. But $\mathcal{T}_{N, c}$ is already quasi-uniform in $\left(\Omega_{c}, I\right)$. Thus it is equivalent to asking that

$$
J^{T} G J=c I \quad \text { or } \quad J^{-T} G^{-1} J^{-1}=c I .
$$

In general we may not get the identity since it is equivalent to asking the manifold $\left(\Omega_{c}, G_{c}\right)$ be flat. We try to approximate this identity as well as possible. 
3.1. An optimization problem in terms of $x=x(\xi)$. Let us first obtain a new optimization problem in terms of $x=x(\xi)$. Let $\left\{\mu_{i}\right\}_{i=1}^{n}$ be the eigenvalues of $G_{c}$. Note that $\mu_{i}>0$ since $G$ is a metric. Following [46], we begin our derivation with the elementary inequality

$$
\frac{1}{n} \sum_{i=1}^{n} \mu_{i} \geq\left(\prod_{i=1}^{n} \mu_{i}\right)^{1 / n}
$$

Therefore,

$$
\frac{1}{n} \operatorname{trace}\left(G_{c}\right) \geq\left(\operatorname{det} G_{c}\right)^{1 / n} .
$$

The matrix $G_{c}$ is a scalar matrix if and only if the above equality holds. Namely the ratio on the left-hand side is minimized.

But

$$
\operatorname{trace}\left(G_{c}\right)=\sum_{i=1}^{m}\left[\left(\nabla x_{i}\right)^{t} G \nabla x_{i}\right], \quad\left(\operatorname{det} G_{c}\right)=(\operatorname{det} J)^{2}(\operatorname{det} G)
$$

thus

$$
\sum_{i=1}^{m}\left(\nabla x_{i}\right)^{t} G \nabla x_{i} \geq n\left[(\operatorname{det} J)^{2}(\operatorname{det} G)\right]^{1 / n} .
$$

Raising this inequality with power $q>n / 2$ and integrating the result on $\Omega_{c}$ gives

$$
\int_{\Omega_{c}}\left(\sum_{i=1}^{n}\left(\nabla x_{i}\right)^{t} G \nabla x_{i}\right)^{q}(\xi) d \xi \geq n^{q} \int_{\Omega_{c}}\left[(\operatorname{det} J)^{2}(\operatorname{det} G)\right]^{q / n}(\xi) d \xi .
$$

By the Hölder inequality,

$$
|\Omega|^{1-n / 2 q}\left\{\int_{\Omega_{c}}\left[(\operatorname{det} J)^{2}(\operatorname{det} G)\right]^{q / n}(\xi) d \xi\right\}^{n / 2 q} \geq \int_{\Omega_{c}}(\operatorname{det} J)(\operatorname{det} G)^{1 / 2}(\xi) d \xi .
$$

Namely

$$
\begin{aligned}
\int_{\Omega_{c}}\left[(\operatorname{det} J)^{2}(\operatorname{det} G)\right]^{q / n}(\xi) d \xi & \geq\left|\Omega_{c}\right|^{1-2 q / n}\left[\int_{\Omega_{c}}(\operatorname{det} J)(\operatorname{det} G)^{1 / 2}(\xi) d \xi\right]^{2 q / n} \\
& =\left|\Omega_{c}\right|^{1-2 q / n}\left[\int_{\Omega}(\operatorname{det} G)^{1 / 2}(x) d x\right]^{2 q / n} \\
& =\left|\Omega_{c}\right|^{1-2 q / n}|\Omega|_{G}^{2 q / n} .
\end{aligned}
$$

In summary, we have

$$
\begin{aligned}
\int_{\Omega_{c}}\left(\sum_{i=1}^{n}\left(\nabla x_{i}\right)^{t} G \nabla x_{i}\right)^{q}(\xi) d \xi & \geq n^{q} \int_{\Omega_{c}}\left[(\operatorname{det} J)^{2}(\operatorname{det} G)\right]^{q / n}(\xi) d \xi \\
& \geq n^{q}\left|\Omega_{c}\right|^{1-2 q / n}|\Omega|_{G}^{2 q / n} .
\end{aligned}
$$

By the well-known properties of two inequalities used above, we note that the first inequality holds with equality if and only if $G_{c}$ has all equal eigenvalues (isotropic), namely $G_{c}$ is a scalar matrix, and the second inequality holds with equality if and only if $\left(\operatorname{det} G_{c}\right)^{1 / 2}=(\operatorname{det} J)(\operatorname{det} G)^{1 / 2}=$ constant (equidistribution). Thus both 
inequalities hold with equalities if and only if both isotropy and equidistribution conditions are satisfied. This argument leads to the optimization problem:

$$
\min _{x} \int_{\Omega_{c}}\left[\sum_{i=1}^{n}\left(\nabla x_{i}\right)^{t} G(x) \nabla x_{i}\right]^{q}(\xi) d \xi, \quad q>n / 2 .
$$

For a given $q>n / 2$, the minimizer of the above functionals is expected to satisfy both equidistribution and isotropy conditions simultaneously. Since the lower bound in the derivation may not be attainable, minimizers for a different $q$ may be different. The index $q$ will control the mesh density. A larger $q$ will lead to a more equidistributed grid.

3.2. An optimization problem in terms of $\xi=\xi(x)$. A similar optimization problem can also be obtained in terms of $\xi=\xi(x)$. From a computational point of view, $x=x(\xi)$ is the transformation that we need to use, but, as we shall explain later, there are some advantages of using its inverse transformation to define the optimization problem.

We can apply similar arguments for the map $\xi=\xi(x)$. Let us assume $J$ is nonsingular. Note that $\left(G_{c}\right)^{-1}=J^{-t} G^{-1} J^{-1}$. Similar to (3.1), we have

$$
\left(\sum_{i=1}^{n} \nabla \xi_{i}^{t} G^{-1} \nabla \xi_{i}\right] \geq n\left[(\operatorname{det} J)^{2}(\operatorname{det} G)\right]^{-1 / n}
$$

and for $q>n / 2$

$$
\int_{\Omega}(\operatorname{det} G)^{1 / 2}\left(\sum_{i=1}^{n} \nabla \xi_{i}^{t} G \nabla \xi_{i}\right)^{q}(x) d x \geq n^{q} \int_{\Omega}\left[(\operatorname{det} J)^{2}(\operatorname{det} G)\right]^{-q / n}(x) d x .
$$

Let $r=2 q / n$ and $r^{\prime}$ be its congruent index; i.e., $1 / r+1 / r^{\prime}=1$. By the Hölder inequality,

$$
\left\|[(\operatorname{det} J)(\operatorname{det} G)]^{-\frac{1}{2 r^{\prime}}}\right\|_{L^{r}}\left\|(\operatorname{det} G)^{\frac{1}{2 r^{\prime}}}\right\|_{L^{r^{\prime}}} \geq \int_{\Omega}(\operatorname{det} J)^{-1}(x) d x=\int_{\Omega_{c}} 1 d \xi=\left|\Omega_{c}\right| .
$$

The equality holds if and only if $(\operatorname{det} J)(\operatorname{det} G)^{1 / 2}=$ constant which is equivalent to the equidistribution condition.

Noting that $\left\|(\operatorname{det} G)^{\frac{1}{2 r^{\prime}}}\right\|_{L^{r^{\prime}}}=\int_{\Omega}(\operatorname{det} G)^{1 / 2} d x=$ constant, we then end up with the optimization problem

$$
\min _{\xi} \int_{\Omega}[(\operatorname{det} G)(x)]^{1 / 2}\left[\sum_{i=1}^{n}\left(\nabla \xi_{i}\right)^{t} G^{-1}(x) \nabla \xi_{i}\right]^{q} d x, \quad q>n / 2 .
$$

Remark 3.1. Monitor functions. The matrix $G(x)$ used in (3.2) and (3.3) is called monitor function in the literature. It is widely used in the existing variational mesh adaptation methods; e.g., see [50, 39, 20, 48, 19, 84. Based on our discussions above, the following choice of monitor function is evident:

$$
G(x)=(\operatorname{det} H)(x)^{-\frac{1}{2 p+n}} H(x) .
$$

3.3. On the minimization problems $(\underline{3.2})$ and $(\underline{3.3})$. Followins are some brief discussions on the minimization problems (3.2) and (3.3). The discussion for the one dimensional case is easy. The situation for multiple dimensions is more complicated. One main conclusion for multiple dimensions is that our optimization problem is significantly different from the harmonic mapping approach which is what has been used most widely in the literature. 
3.3.1. One dimension $n=1$. In one dimension, namely $n=1$, as we shall see now, both optimization problems can be solved exactly and in fact the solutions to both problems are identical to each other. The result we obtain in one dimension is not new and it coincides, for example, with the so-called grading function obtained by Carey and Dinh 22 .

Without loss of generality, we may suppose that $\Omega=(0,1)$. Solving functional (3.2) gives us

$$
\left(G^{q} x^{\prime 2 q}\right)^{\prime}=0 \Rightarrow G^{1 / 2}(x) x^{\prime}(\xi)=c \Rightarrow \xi^{\prime}(x)=c^{-1} G^{1 / 2}(x) .
$$

We obtain

$$
\xi(x)=\frac{\int_{0}^{x} G^{1 / 2}(t) d t}{\int_{0}^{1} G^{1 / 2}(t) d t},
$$

which will be called the mesh distribution function. The asymptotic optimal mesh can be obtained by the inverse of the mesh distribution function, more precisely $x_{i}=$ $\xi^{-1}\left(\xi_{i}\right), i=0,1, \ldots, N, \xi_{i}=i / N$, which may be obtained by the Newton method or a discrete version of the mesh distribution function. Recently the mesh distribution function has been applied successfully to the polygonal curve simplification [27.

Now, we consider the optimization problem (3.3). In this case, the EulerLagrangian equation becomes

$$
\left(G^{1 / 2-q} \xi^{\prime}(x)^{2 q-1}\right)^{\prime}=0
$$

which gives

$$
\xi^{\prime}(x)=c G^{1 / 2}(x) .
$$

This is the same as (3.5). Thus we get the same mesh distribution function from (3.3).

We summarize the above discussions as follows.

Proposition 3.2. In one dimension, both optimization problems (3.2) and (3.3) are equivalent and their minimizer, known as the mesh distribution function, is given by (3.6), which is independent of the parameter $q$.

3.3.2. Multiple dimensions. The situations in multiple dimensions are much more complex. Let us first point out that the optimization problem (3.3) appears to have more desirable properties than (3.2). For example, the functional in (3.3) is strictly convex while the functional in (3.2) is more complex. Especially, for $q=1$, the Euler-Lagrange equation for (3.3) is linear while the Euler-Lagrange equation for (3.2) is always nonlinear.

Since the problem (3.3) is strictly convex, the existence and uniqueness of the minimizer is then obvious for this problem. A less obvious question is if the minimizer $\xi=\xi(x)$ is actually a homeomorphism between $\Omega$ and $\Omega_{c}$ and furthermore if its Jacobian is nonsingular. These two questions are already well studied for the special case that $q=1$ and $n=2$ in the literature and the answers to both questions are affirmative under the assumption that the computational domain $\Omega_{c}$ is convex (see [44, 78]). One natural question to ask is whether these results can also be extended to the case that $q>1$. This question is significant especially for $n \geq 3$ and it will be a subject of further study.

The optimization problem (3.2), on the other hand, is more complex to analyze than (3.3). Let us now only discuss the existence for this problem as the answers to other relevant questions are not so obvious. 
Theorem 3.3. Given a homeomorphism $g: \partial \Omega_{c} \mapsto \partial \Omega$, there is a transformation $x: \Omega_{c} \rightarrow \Omega$ such that it is a minimizer of the minimization problem (3.2) subject to

$$
x \in\left(W^{1,2 q}\left(\Omega_{c}\right)\right)^{n}, \quad x(\xi)=g(\xi), \quad \xi \in \partial \Omega_{c} .
$$

This theorem can be established by using the following simple lemma.

Lemma 3.4. Assume that $G \in\left(L^{\infty}(\Omega)\right)^{n \times n}$, is continuous almost everywhere in $\Omega^{n \times n}$, and is uniformly symmetric positive definite in $\Omega$. Then

$$
\int_{\Omega_{c}}\left[\sum_{i=1}^{n}\left(\nabla x_{i}\right)^{t} G(x) \nabla x_{i}\right]^{q}(\xi) d \xi
$$

is lower semi-continuous in $\left(W^{1,2 q}\left(\Omega_{c}\right)\right)^{n}$ for a given $q>n / 2$.

Proof. Let us first assume that $G$ is continuous everywhere in $\Omega^{n \times n}$, namely $G \in$ $\left(C_{B}(\Omega)\right)^{n \times n}$. When $q>n / 2, W^{1,2 q}\left(\left(\Omega_{c}\right)^{n}\right)$ is compactly embedded in $\left(L^{\infty}\left(\Omega_{c}\right)\right)^{n}$. Thus if $\left(x^{m}\right)$ is a bounded sequence in $\left(W^{1,2 q}\left(\Omega_{c}\right)\right)^{n}$ that is weakly convergent to $x^{*}$, we have $x^{m} \rightarrow x^{*}$ strongly in $\left(C\left(\Omega_{c}\right)\right)^{n}$. By a simple mean value theorem:

$$
\begin{aligned}
\mid \int_{\Omega_{c}}[ & \left.\sum_{i=1}^{n}\left(\nabla x_{i}^{m}\right)^{t} G\left(x^{m}\right) \nabla x_{i}^{m}\right]^{q}(\xi) d \xi-\int_{\Omega_{c}}\left[\sum_{i=1}^{n}\left(\nabla x_{i}^{m}\right)^{t} G\left(x^{*}\right) \nabla x_{i}^{m}\right]^{q}(\xi) d \xi \mid \\
= & q \int_{\Omega_{c}}\left\{\sum_{i=1}^{n}\left(\nabla x_{i}^{m}\right)^{t}\left[\theta G\left(x^{m}\right)+(1-\theta) G\left(x^{m}\right)\right] \nabla x_{i}^{m}\right\}^{q-1} \\
& \cdot\left\{\sum_{i=1}^{n}\left(\nabla x_{i}^{m}\right)^{t}\left[G\left(x^{m}\right)-G\left(x^{*}\right)\right] \nabla x_{i}^{m}\right\}(\xi) d \xi \\
\leq & q\|G\|_{L^{\infty}}\left\|G\left(x^{m}\right)-G\left(x^{*}\right)\right\|_{L^{\infty}} \int_{\Omega_{c}} \sum_{i}\left|\nabla x_{i}^{m}\right|^{2 q}(\xi) d \xi .
\end{aligned}
$$

It follows that

$$
\begin{aligned}
& \lim _{m \rightarrow \infty} \inf \int_{\Omega_{c}}\left[\sum_{i=1}^{n}\left(\nabla x_{i}^{m}\right)^{t} G\left(x^{m}\right) \nabla x_{i}^{m}\right]^{q}(\xi) d \xi \\
= & \lim _{m \rightarrow \infty} \inf \int_{\Omega_{c}}\left[\sum_{i=1}^{n}\left(\nabla x_{i}^{m}\right)^{t} G\left(x^{*}\right) \nabla x_{i}^{m}\right]^{q}(\xi) d \xi \\
\geq & \int_{\Omega_{c}}\left[\sum_{i=1}^{n}\left(\nabla x_{i}^{*}\right)^{t} G\left(x^{*}\right) \nabla x_{i}^{*}\right]^{q}(\xi) d \xi
\end{aligned}
$$

where, in the last step, we have used the fact that

$$
\int_{\Omega_{c}}\left[\sum_{i=1}^{n}\left(\nabla x_{i}\right)^{t} G\left(x^{*}\right) \nabla x_{i}\right]^{q}(\xi) d \xi
$$

is semicontinuous as a convex nonlinear functional of $x$. This completes the lemma when $G \in\left(C_{B}(\Omega)\right)^{n \times n}$. For the general case $G \in\left(L^{\infty}(\Omega)\right)^{n \times n}$, the result follows from the standard density argument. 
3.3.3. Comparisons with harmonic mapping. Theories based on harmonic mapping have been used extensively in formulating variational mesh generation techniques. The advantages of this approach include

- It is easy to get the existence and uniqueness of the minimizer.

- The Euler-Lagrange equation of this functional is a linear elliptic equation which is easy to solve.

For a comprehensive treatment, we refer to the book 64 and the references therein. Here we only point out that it corresponds to $q=1$ case in (3.3). Based on the requirement $q>n / 2$, we see for one dimension, the harmonic mapping approach is justified by our theory. But for multiple dimensions, it seems that the harmonic mapping only addresses the isotropy property not the equidistribution property. We also note that $n=2$ is the borderline case for $q>n / 2$ when harmonic mapping $(q=1)$ is used. Hence the harmonic mapping may still be a reasonable approach in two dimensions and it may not be the case for $n \geq 3$.

3.4. Monitor functions for nonuniform computational meshes. The optimization problems have been obtained based on the assumption that the computational grid is quasi-uniform (with respect to the usual Euclidean metric). In this section, we propose a slight modification of the monitor function to relax the quasiuniform assumption on the computational domain. This modification is useful when the moving mesh method is combined with the local grid refinement method.

Given a computational grid $\mathcal{T}_{N, c}$ which may not be quasi-uniform but is assumed to be shape regular (namely the all the elements are locally isotropic), we introduce a size function $s(\xi)=\left|\tau_{c}(\xi)\right|^{2 / n}$, where $\tau_{c}(\xi)$ means the element in $\mathcal{T}_{N, c}$ which contains point $\xi$. Noting that $s(\xi)$ is a scalar function, if we use

$$
G(x)=s(\xi(x))[(\operatorname{det} H)(x)]^{-\frac{1}{2 p+n}} H(x)
$$

in (3.2) or (3.3), the isotropy condition is still satisfied for the minimizer of (3.2) or (3.3). Meanwhile the minimizer is aimed to satisfy

$$
s^{n}(\operatorname{det} J)^{2}(\operatorname{det} G)=\left(\operatorname{det} G_{c}\right)\left|\tau_{c}\right|^{2}=\left|\tau_{c}\right|_{G_{c}}^{2}=\text { constant, }
$$

which means the triangulation satisfies the equidistribution condition.

By using this modified metric, we can use a shape-regular mesh in the computational domain. Conventional isotropic refinement strategies, namely regular division [11, 12, 10] and bisection [79, 76, 75], will result in a regular mesh. Thus it is possible to combine moving mesh ( $r$-type adaptive method) and local refinement ( $h$-type adaptive method) together to get a more efficient algorithm to solve equations. A combination of those two methods has been studied in 11, 8, 42, 21].

\section{Numerical EXPERIMENTS}

In this section, we shall report some numerical examples that support the interpolation error estimates presented in Section 2. We are not going to discuss all the details of our algorithm that produce the underlying meshes since this paper mainly concentrates on theoretical issues of interpolation errors. For the description of our algorithm, see our recent work [26, 24]. More sophisticated algorithms for a Delaunay-type mesh generation algorithm governed by Riemannian metrics can be found in [17, 18, 
TABLE 1. Errors for the one dimensional example

\begin{tabular}{|c|c|c|}
\hline$N$ & Error & $N^{2} \times$ Error \\
\hline 32 & $2.9756 e-004$ & $3.0470 e-001$ \\
\hline 64 & $7.4198 e-005$ & $3.0392 e-001$ \\
\hline 128 & $1.8519 e-005$ & $3.0342 e-001$ \\
\hline 256 & $4.6285 e-006$ & $3.0333 e-001$ \\
\hline 512 & $1.1570 e-006$ & $3.0331 e-001$ \\
\hline 1024 & $2.8925 e-007$ & $3.0330 e-001$ \\
\hline
\end{tabular}

4.1. One dimensional example. We first report some tests in the one dimensional case. We choose the test function

$$
u=e^{-\frac{x}{\epsilon}}+0.5 x^{2}, \quad x \in[0,1],
$$

with $\epsilon=10^{-3}$. This function has a boundary layer near $x=0$. We combine refinement and local moving strategies to get the optimal mesh for this function. The ideal convergent rate is $N^{-2}$ if we choose a (nearly) optimal mesh, where $N$ is the number of intervals. We begin with $N=32$, using the mesh distribution function to move mesh points to get a good initial mesh in this coarse level, and then do the refinement. Once we insert a point into an interval, we move this new point in this interval so that it is the middle point of this interval under the new metric.

Table 1 contains the results of computation on six optimal meshes for the $L^{2}$ norm. In the last column, we compute $N^{2} \times$ Error to show that we achieve the optimal convergent rate. Figure 1 also shows the convergent rate clearly.

Note that in one dimension, we only have the equidistribution condition. To show our mesh satisfies this condition, we computed the error on each interval for the finest mesh $N=1024$ and the sorted errors are shown in Figure 2. From this picture we can see that $96 \%$ of errors are almost the same, which illustrates the equidistribution principle.

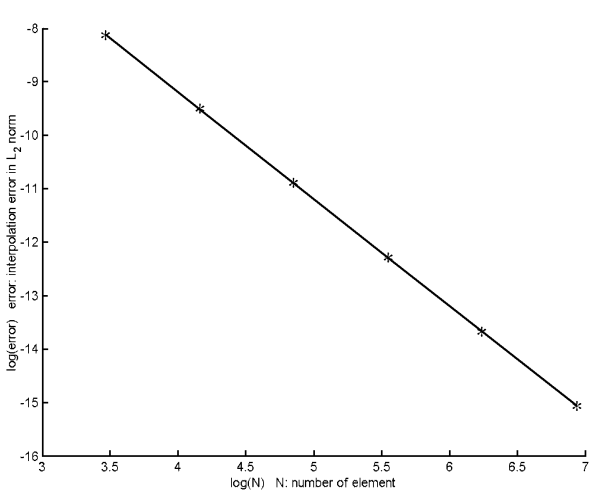

FIGURE 1. Convergent rate

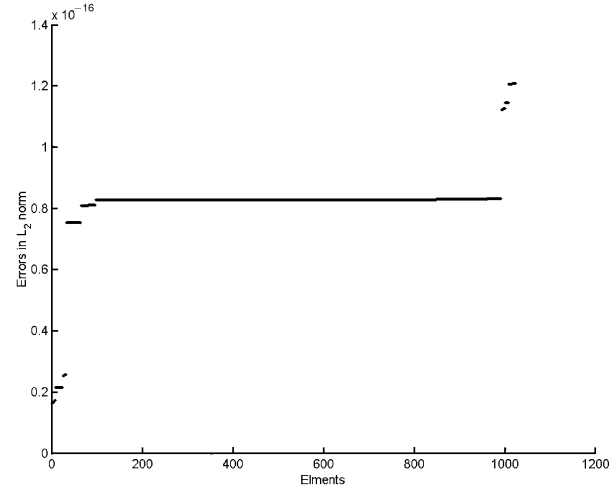

FIGURE 2. Distribution of the error 


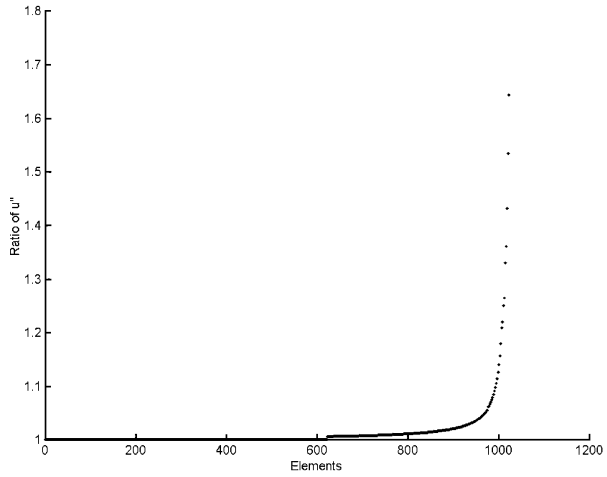

(a) Assumption (A1)

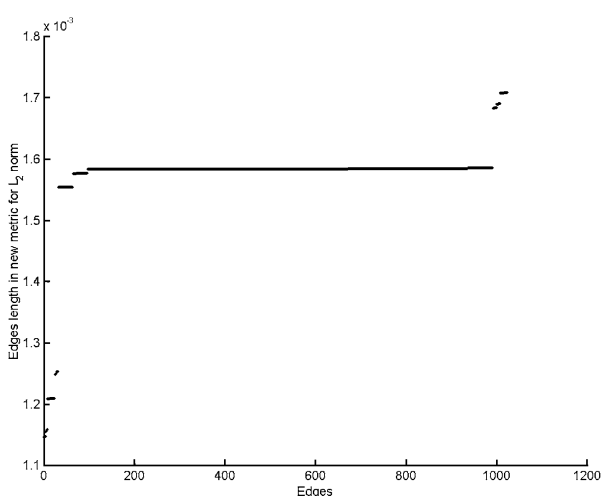

(b) Assumption (A2)

Figure 3. Validation of assumptions (A1) and (A2)

To show that assumptions (A1) and (A2) are satisfied, we plot edge lengths under the new metric and the ratio of $u^{\prime \prime}$ in each interval. Since $u^{\prime \prime}$ is monotone decreasing in $[0,1]$, we compute $r(i)=\left|u^{\prime \prime}(i) / u^{\prime \prime}(i+1)\right|$. Figure 3(a) shows that $99 \%$ of $r(i)$ are below 1.1 and the maximum ratio is 1.64 and Figure 3(b) shows that $96 \%$ of edge lengths are almost the same and it varies from $1.15 e-3$ to $1.71 e-3$. All the data in Figure 3 is sorted.

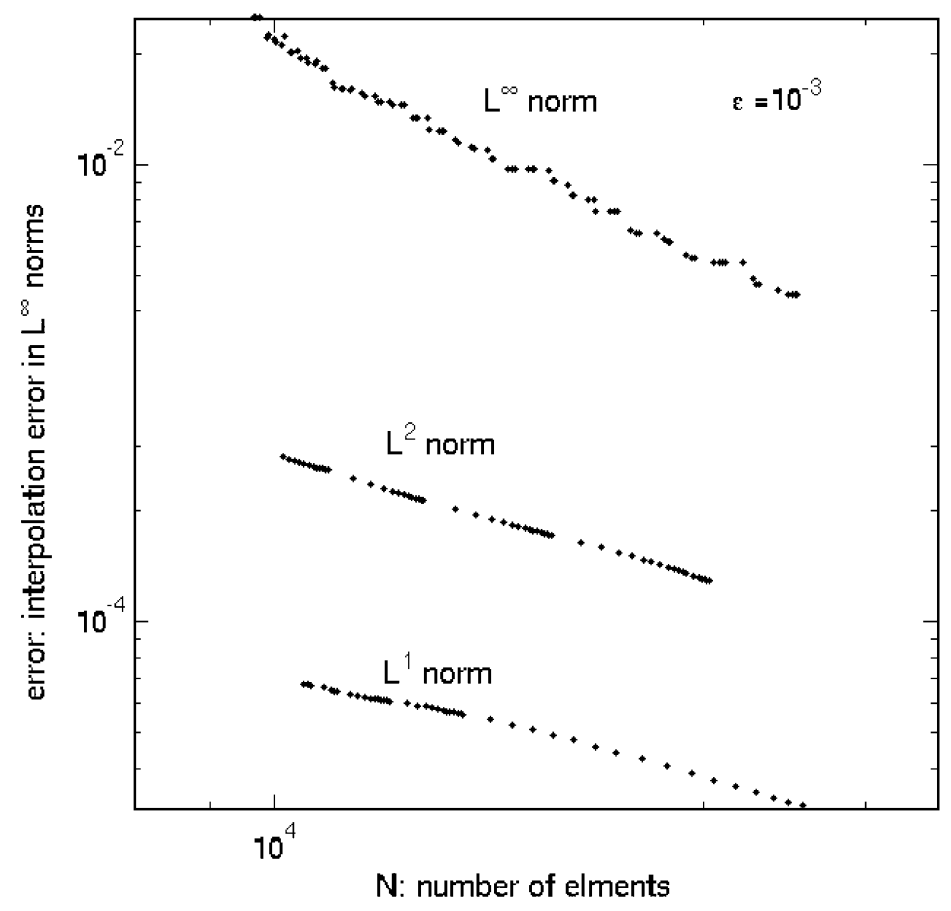

Figure 4. Optimal convergent rate in different norms 


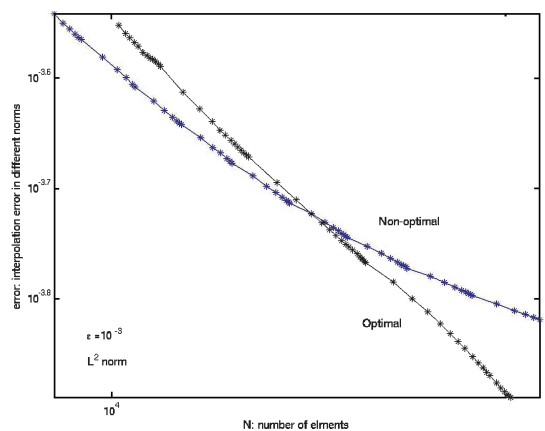

(a) for $L^{1}$ norm

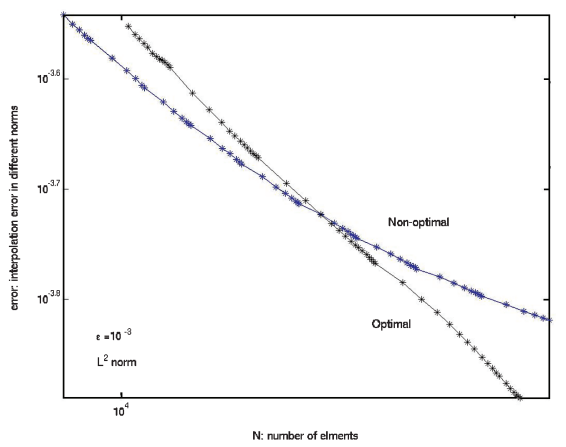

(b) for $L^{2}$ norm

Figure 5. Optimal mesh vs. Non-optimal mesh

4.2. Two dimensional examples. In two dimensions, we use the test function

$$
u(x, y)=e^{-\left(\frac{r-0.5}{\epsilon}\right)^{2}}+0.5 r^{2},
$$

where $r^{2}=(x+0.1)^{2}+(y+0.1)^{2}$. This function changes dramatically at the $\epsilon$ neighborhood of $r=0.5$. We use the offset $(x+0.1, y+0.1)$ to avoid the nonsmoothing Hessian matrix at $(0,0)$ and quadratic function $0.5 r^{2}$ to ensure the Hessian matrix is not singular when $r$ is far away from the shock so we can focus our attention near the shock only.

We first test the theoretical convergent rate by fixing $\epsilon=10^{-3}$. We measured the error in the $L_{\infty}, L_{2}, L_{1}$ norms. Then the algorithm will try to produce equilateral triangles in different metrics and the ideal convergent rate will be $N^{-1}$ using optimal meshes, where $N$ is the number of triangles. Since we refine and move the mesh many times (from $N \approx 8000$ to $N \approx 20000$ ), we are not going to list the error table

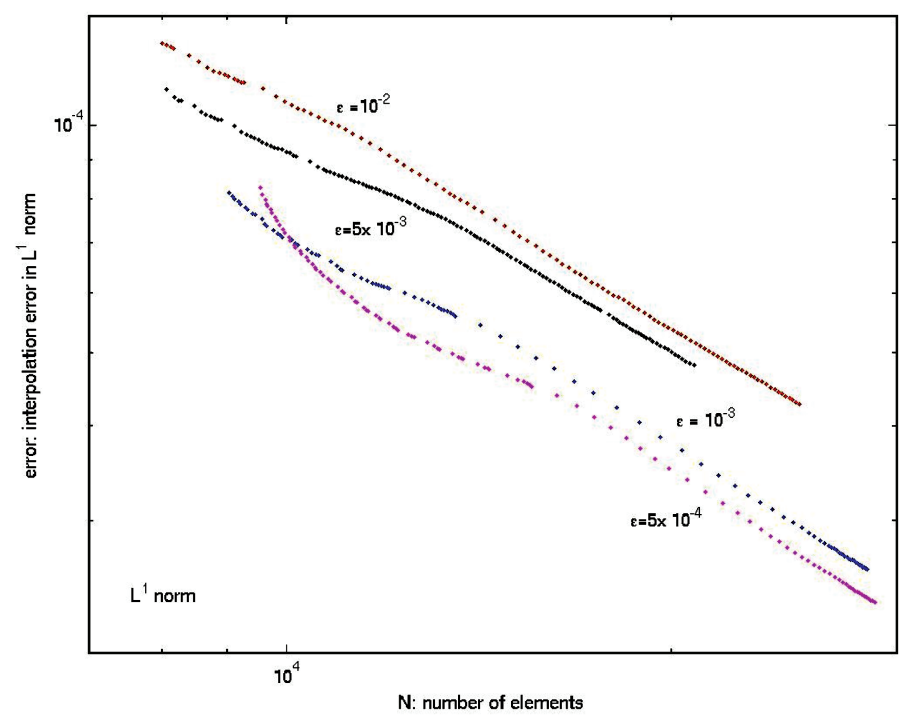

FiguRE 6. Uniformly bounded with respect to $\epsilon$ 


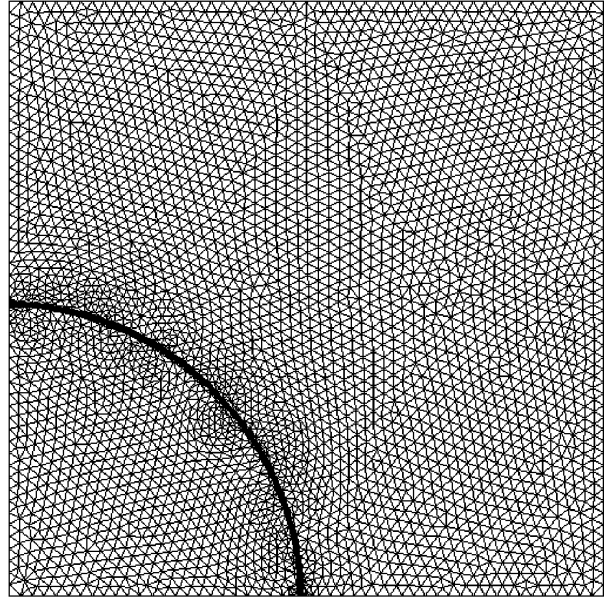

(a) Mesh 1

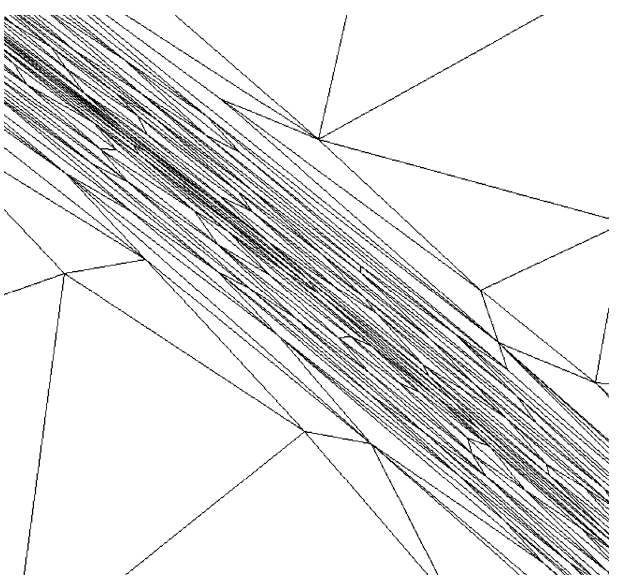

(c) Mesh 3

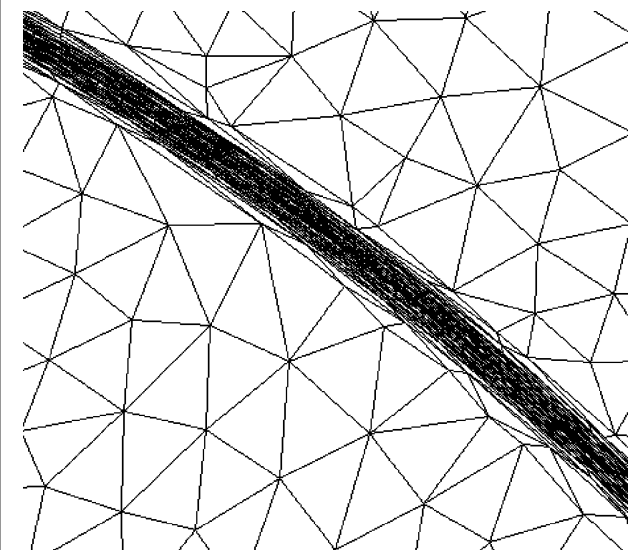

(b) Mesh 2

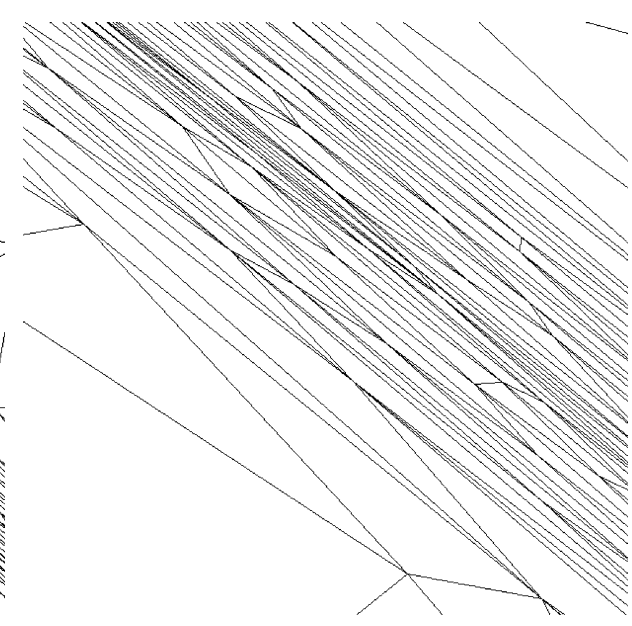

(d) Mesh 4

FiguRE 7. An anisotropic mesh and its details

at each time. Figure 4 shows the convergent rate for $L_{\infty}, L_{2}, L_{1}$. Note that the constant to which $N \times$ Error converges is monotone increasing with respect to $p$.

To show the optimality of our scaling metric, we also compute the suboptimal mesh by only using the Hessian matrix. Since the scaling $\mu_{p}$ only affects the mesh density (equidistribution), its advantage appears when the number of elements is bigger; see Figure 5 .

Now we fix the error norm and let $\epsilon$ vary. As in the error bound, $\|\operatorname{det} H\|_{\frac{p}{p+1}}$ is bounded independently of $\epsilon$, which means if we get the optimal mesh, the convergence rate is independent of $\epsilon$. Indeed it will actually get better when $\epsilon$ is smaller (since in this case $\|\operatorname{det} H\|_{\frac{p}{p+1}}=O\left(\epsilon^{1 /(p+1)}\right)$. Figure 6] illustrates this observation.

It is clear that the function only changes rapidly in the $r$ direction around the shock if we use polar coordinates. Thus the mesh around the shock is aligned to the shock, namely the edges are large along the tangential direction of circle $r=0.5$ while they are small along the normed direction. In the remaining part of 
the region, the metric is dominated by the quadratic part. Thus they are nearly equilateral triangles when they are away from the shock. In Figure 7 we present several pictures of our meshes.

\section{COnClusion And FUture WORK}

In this paper, we have obtained optimal interpolation error estimates in the $L^{p}$ norm and, based on the estimate, we have derived new criteria for optimal meshes that minimize the $L^{p}$ error. We further use the estimate to derive some new optimization problem for the global moving mesh method. This optimization problem is different from the harmonic function approach used in the literature. In particular, we present an optimization problem that can also be used when the computational mesh is not uniform (hence the global mesh moving and local mesh refinement can be combined).

We have, however, not discussed much how these theoretical results can be used in adaptive finite element approximations to partial differential equations. To carry out the interpolation results to finite element approximations, there are many questions that need to be answered.

One such question is how the anisotropic mesh can be practically generated to satisfy the assumptions required in our theorems. Of course, many existing techniques in the literature can be used for this but some special issues need also to be addressed to satisfy the special requirements from our new theoretical results. We have indeed developed all relevant techniques including new techniques for refinement, coarsening, edge swapping, and local smoothing to improve the mesh quality. We refer to our recent works [26, 28, 24, for details.

Another critical question that needs to be answered is how the Hessian matrix of the solution can be obtained when linear finite element approximation is used for the discretization of partial differential equations. Since taking piecewise second derivatives to piecewise linear functions will not lead to any useful approximation to the Hessian matrix, special postprocessing techniques need to be used to obtain reasonable Hessian matrix approximation from linear finite elements. Fortunately, there have been many techniques developed in the literature for superconvergent recovery of the gradient of the solution, which would naturally lead to good recovery schemes for the Hessian matrix. On superconvergent recovery schemes, we refer to Zikienwicz-Zhu [87, 86, Hoffmann-Schatz-Wahlbin-Wittum [45], Zhang-Naga 85, Bank-Xu [14, 13, and $\mathrm{Xu}$-Zhang 82, etc. We note, however, that all these results were derived for isotropic elements. How to generalize these results or to develop new techniques for anisotropic meshes is a problem for further research. We have in fact some preliminary results in this direction, but this will be a topic for another paper.

In addition to the above questions, we are also very interested in applying our results to problems such as convection-dominated problems. In 26] we observed that a properly adapted mesh will enhance the stability of the standard finite element methods which often fails for convection dominated problems on quasiuniform grids. This phenomenon has been observed in other simpler situations (see books Miller et al. [68, Roos et al. [77]). In the current work, the mesh is adapted to optimize the interpolation error. We expect that the discretization 
error will inherit the optimality of the interpolation error on a nearly optimal mesh for the linear interpolant. We have obtained some preliminary results for a one dimensional convection dominated model problem. In [29, 30] we show that for a carefully designed streamline diffusion finite element method the discretization error is controlled by the interpolation error (in the $L^{\infty}$ norm).

\section{REFERENCES}

[1] S. Adjerid and J. E. Flaherty. A moving-mesh finite element method with local refinement for parabolic partial differential equations. Comp. Meht. Appl. Mech. Engry., 55:3-26, 1986. MR0845411 (87h:65187)

[2] A. Agouzal, K. Lipnikov, and Y. Vassilevski. Adaptive generation of quasi-optimal tetrahedral meshes. East-West J. Numer. Math., 7:223-244, 1999. MR.1738437 (2000i:65204)

[3] A. Agouzal and Y. Vassilevski. On a discrete Hessian recovery for $P_{1}$ finite elements. $J$. Numer. Math., 10:1-12, 2002. MR.1905846 (2003c:65137)

[4] T. Apel. Anisotropic finite elements: Local estimates and applications. Book Series: Advances in Numerical Mathematics. Stuttgart: Teubner, 1999. MR.1716824 (2000k:65002)

[5] T. Apel, M. Berzins, P. K. Jimack, G. Kunert, A. Plake, I. Tsukerman, and M. Walkley. Mesh shape and anisotropic elements: Theory and practice. In J. R. Whiteman (ed. ): The Mathematics of Finite Elements and Applications X, Elsevier, Amsterdam,, pages 367-376, 2000. MR1801989 (2001j:65180)

[6] T. Apel, S. Grosman, P. K. Jimack, and A. Meyer. A new methodology for anisotropic mesh refinement based upon error gradients. Appl. Numer. Math., 50(3-4):329-341, 2004. MR2074008

[7] T. Apel and J. Schoberl. Multigrid methods for anisotropic edge refinement. SIAM Journal on Numerical Analysis, 40(5):1993-2006, 2000. MR1950630(2003m:65225)

[8] D. C. Arney and J. E. Flaherty. An adaptive mesh-moving and local refinement method for time-dependentt partial differential equatioins. ACM Trans. Math. Software, 16:48-71, 1990. MR.1073410 (91f:65154)

[9] I. Babuška and W. C. Rheinboldt. Error estimates for adaptive finite element computations. SIAM Journal on Numerical Analysis, 15:736-754, 1978. MR0483395 (58:3400)

[10] I. Babuška and T. Strouboulis. The Finite Element Method and Its Reliability. Numerical Mathematics and Scientific Computation, Oxford Science Publications, 2001. MR1857191 (2002k:65001)

[11] R. E. Bank and A. H. Sherman. An adaptive multilevel method for elliptic boundary value problems. Computing, 26:91-105, 1981. MR0619932 (83c:65229)

[12] R. E. Bank, A. H. Sherman, and A. Weiser. Refinement algorithms and data structures for regular local mesh refinement. In R. S. et al., editor, Scientific Computing, pages 3-17. IMACS/North-Holland Publishing Company, Amsterdam, 1983. MR0751598

[13] R. E. Bank and J. Xu. Asymptotically exact a posteriori error estimators, Part I: Grids with superconvergence. SIAM Journal on Numerical Analysis, 41(6):2294-2312, 2003. MR2034616 (2004k:65194)

[14] R. E. Bank and J. Xu. Asymptotically exact a posteriori error estimators, Part II: General unstructured grids. SIAM Journal on Numerical Analysis, 41(6):2313-2332, 2003. MR2034617 (2004m:65212)

[15] G. Beckett, J. A. Mackenzie, A. Ramage, and D. M. Sloan. Computational solution of twodimensional unsteady PDEs using moving mesh methods. J. Comput. Phys., 182(2):478-495, November 2002. MR.1941849 (2003m:65169)

[16] H. Borouchaki, M. J. Castro-Diaz, P. L. George, F. Hecht, and B. Mohammadi. Anisotropic adaptive mesh generation in two dimensions for CFD. In 5th International Conference On Numerical Grid Generation in Computational Field Simulations, volume 3, pages 197-206. Mississppi State University, 1996.

[17] H. Borouchaki, P. L. George, F. Hecht, P. Laug, and E. Saltel. Delaunay mesh generation governed by metric specifications. I. algorithms. Finite Elem. Anal. Des., 25(1-2):61-83, 1997. MR:1442300 (98c:65163) 
[18] H. Borouchaki, P. L. George, and B. Mohammadi. Delaunay mesh generation governed by metric specifications. II. applications. Finite Elem. Anal. Des., 25(1-2):85-109, 1997. MR.1442301 (98c:65164)

[19] J. U. Brackbill and J. S. Saltzman. Adaptive zoning for singular problems in two dimensions. J. Comput. Phys., 46:342-368, 1982. MR0673707 (84c:65140)

[20] W. Cao, W. Huang, and R. D. Russell. A study of monitor functions for two dimensional adaptive mesh generation. SIAM J. Sci. Comput., 20:1978-1994, 1999. MR.1694650|(2000c:65113)

[21] W. Cao, J. Lang, W. Huang, and R. D. Russell. A two-dimensional moving finite element method with local refinement based on a posteriori error estimates. Appl. Numer. Math., 46:75-94, 2003. MR:1986348(2004d:65113)

[22] G. F. Carey and H. T. Dinh. Grading functions and mesh redistribution. SIAM Journal on Numerical Analysis, 22(5):1028-1040, 1985. MR0799126 (86h:65123)

[23] H. D. Ceniceros and T. Y. Hou. An efficient dynamically adaptive mesh for potentially singular solutions. Journal of Computational Physics, 172:609-639, 2001.

[24] L. Chen. Mesh smoothing schemes based on optimal Delaunay triangulations. In 13th International Meshing Roundtable, pages 109-120, Williamsburg, VA, 2004. Sandia National Laboratories.

[25] L. Chen. New analysis of the sphere covering problems and optimal polytope approximation of convex bodies. Journal of Approximation Theory, 133(1):134-145, March 2005. MR2122271 (2005k:41097)

[26] L. Chen, P. Sun, and J. Xu. Multilevel homotopic adaptive finite element methods for convection dominated problems. In The Proceedings for 15th Conferences for Domain Decomposition Methods, Lecture Notes in Computational Science and Engineering 40, pages 459-468. Springer, 2004.

[27] L. Chen, J. Z. Wang, and J. Xu. Asymptotically optimal and linear-time algorithm for polygonal curve simplification. Submitted to IEEE, 2005.

[28] L. Chen and J. Xu. Optimal Delaunay triangulations. Journal of Computational Mathematics, 22(2):299-308, 2004. MR2058939 (2005c:41042)

[29] L. Chen and J. Xu. An optimal streamline diffusion finite element method for a singularly perturbed problem. In AMS Contemporary Mathematics Series: Recent Advances in Adaptive Computation, volume 383, pages 236-246, Hangzhou, 2005. MR.2195801

[30] L. Chen and J. Xu. Stability and accuracy of adapted finite element methods for singularly perturbed problems. Submitted to Numer. Math., 2005.

[31] E. F. D'Azevedo. Optimal triangular mesh generation by coordinate transformation. SIAM J. Sci. Statist. Comput., 12:755-786, 1991. MR.1102406 (92a:65041)

[32] E. F. D'Azevedo and R. B. Simpson. On optimal interpolation triangle incidences. SIAM J. Sci. Statist. Comput., 6:1063-1075, 1989. MR1025475 (91a:65019)

[33] C. de Boor. Good approximation by splines with variable knots. Int. Seines Numer. Math, Birkhauser Verlag, Basel, 21:57-72, 1973. MR0403169 (53:6982)

[34] C. de Boor. Good approximation by splines with variables knots II. In G. A. Watson, editor, Proceedings of the Eleventh International Conference on Numerical Methods in Fluid Dynamics, volume 363, pages 12-20. Springer-Verlag, Dundee, Scotland, 1974. MR0431606 (55:4603)

[35] Y. Di, R. Li, T. Tang, and P. Zhang. Moving mesh finite element methods for the incompressible Navier-Stokes equations. SIAM J. Sci. Comput., 26(3):1036-1056, 2005. MR2126125 (2005j:65107)

[36] V. Dolejsi. Anisotropic mesh adaptation for finite volume and finite element methods on triangular meshes. Computing and Visualization in Science, 1:165-178, 1998.

[37] V. Dolejsi. Anisotropic mesh adaptation technique for viscous flow simulation. East-West Journal of Numerical Mathematics, 1-24,2001, 9(1):1-24, 2001. MR1839196 (2002c:65164)

[38] T. Dupont and Y. Liu. Symmetric error estimates for moving mesh galerkin methods for advection-diffusion equations. SIAM Journal on Numerical Analysis, 40(3):914-927, 2002. MR1949398 (2004b:65147)

[39] A. S. Dvinsky. Adaptive grid generation from harmonic maps on Riemannian manifolds. J. Comput. Phys., 95(2):450-476, 1991. MR1117849(92e:65162)

[40] L. Formaggia and S. Perotto. New anisotropic a priori error estimates. Numer. Math., 89(4):641-667, 2001. MR1865506 (2002j:65110) 
[41] L. Formaggia and S. Perotto. Anisotropic error estimates for elliptic problems. Numer. Math., pages 67-92, 2003. MR 1971213 (2004c:65127)

[42] W. D. Gropp. Local uniform mesh refinement with moving grids. SIAM J. Sci. Statist. Comput., 8:292-304, 1987. MR0883772 (88f:65161)

[43] W. G. Habashi, M. Fortin, J. Dompierre, M. G. Vallet, D. Ait-Ali-Yahia, Y. Bourgault, M. P. Robichaud, A. Tam, and S. Boivin. Anisotropic mesh optimization for structured and unstructured meshes. In 28th Computational Fluid Dynamics Lecture Series. von Karman Institute, March 1997.

[44] R. Hamilton. Harmonic Maps of Manifolds with Boundary, volume 471 of Lecture Notes in Math. American Mathematical Society, Springer-Verlag, New York, 1975. MR0482822 $(58: 2872)$

[45] W. Hoffmann, A. H. Schatz, L. B. Wahlbin, and G. Wittum. Asymptotically exact a posteriori estimators for the pointwise gradient error on each element in irregular meshes I: A smooth problem and globally quasi-uniform meshes. Mathematics of Computation, 70:897-909, 2001. MR.1826572 (2002a:65178)

[46] W. Huang. Practical aspects of formulation and solution of moving mesh partial differential equations. J. Comput. Phys., 171:753-775, 2001. MR1848733(2002e:65132)

[47] W. Huang. Variational mesh adaptation: isotropy and equidistribution. J. Comput. Phys., 174:903-924, 2001. MR1868106

[48] W. Huang and R. D. Russell. Moving mesh strategy based on a gradient flow equation for twodimensional problems. SIAM J. Sci. Comput., 20:998-1015, 1999. MR1665654 (99m:65179)

[49] W. Huang and W. Sun. Variational mesh adaptation II: Error estimates and monitor functions. J. Comput. Phys., 184:619-648, 2003. MR1959407

[50] P. Knupp. Mesh generation using vector-fields. J. Comput. Phys., 119:142-148, 1995. MR:1336033 (96b:65119)

[51] G. Kunert. A posteriori error estimation for anisotropic tetrahedral and triangular finite element meshes. Ph. D. Thesis, 1999.

[52] G. Kunert. An a posteriori residual error estimator for the finite element method on anisotropic tetrahedral meshes. Numer. Math., 86(3):471-490, 2000. MR1785418 (2001g:65141)

[53] G. Kunert. A local problem error estimator for anisotropic tetrahedral finite element meshes. SIAM Journal on Numerical Analysis, 39(2):668-689, 2001. MR1860258 (2002g:65145)

[54] G. Kunert. A posteriori $l^{2}$ error estimation on anisotropic tetrahedral finite element meshes. IMA Journal of Numerical Analysis, 21(2):503-523, 2001. MR.1825834 (2002a:65162)

[55] G. Kunert. Toward anisotropic mesh construction and error estimation in the finite element method. Numerical Methods for Partial Differential Equations, 18(5):625-648, 2002. MR.1919601 (2003g:65153)

[56] G. Kunert. A posteriori error estimation for convection dominated problems on anisotropic meshes. Math. Meth. Appl. Sci., 26(7):589-617, 2003. MR.1967323 (2004e:65123)

[57] G. Kunert and S. Nicaise. Zienkiewicz-zhu error estimators on anisotropic tetrahedral and triangular finite element meshes. M2AN Math. Model. Numer. Anal., 37(6):1013-1043, 2003. MR2026406 (2004k:65196)

[58] G. Kunert and R. Verfürth. Edge residuals dominate a posteriori error estimates for linear finite element methods on anisotropic triangular and tetrahedral meshes. Numer. Math., 86(2):283-303, 2000. MR 1777490 (2001i:65127)

[59] R. Li, T. Tang, and P. Zhang. Moving mesh methods in multiple dimensions based on harmonic maps. J. Comput. Phys., 170(2):562-588, July 2001. MR.1844903 (2002e:65139)

[60] R. Li, T. Tang, and P. Zhang. A moving mesh finite element algorithm for singular problems in two and three space dimensions. J. Comput. Phys., 177(2):365-393, April 2002. MR1897293 (2003c:65093)

[61] K. Lipnikov and Y. Vassilevski. Optimal triangulations: Existence, approximation and double differentiation of $P_{1}$ finite element functions. Comput. Math. Math. Phys., 43(6):827-835, 2003. MR.1994415 (2004f:65205)

[62] K. Lipnikov and Y. Vassilevski. Parallel adaptive solution of 3d boundary value problems by hessian recovery. Comput. Methods Appl. Mech. Engrg., 192:1495-1513, 2003. MR.1963061

[63] K. Lipnikov and Y. Vassilevski. Error estimates for Hessian-based mesh adaptation algorithms with control of adaptivity. In 13th International Meshing Roundtable, pages 345-351. Sandia National Laboratories, 2004. 
[64] V. D. Liseikin. Grid Generation Methods. Springer Verlag, Berlin, 1999. MR:1707310 (2000d:65001)

[65] Y. Liu, R. E. Bank, T. F. Dupont, S. Garcia, and R. F. Santos. Symmetric error estimates for moving mesh mixed methods for advection-diffusion equations. SIAM Journal on Numerical Analysis, 40(6):2270-2291, 2003. MR.1974185 (2004f:65141)

[66] J. A. Mackenzie and M. L. Robertson. A moving mesh method for the solution of the one-dimensional phase-field equations. J. Comput. Phys., 181(2):526-544, Sept. 2002. MR.1927400 (2003h:65111)

[67] S. Micheletti, S. Perotto, and M. Picasso. Stabilized finite elements on anisotropic meshes :a priori error estimates for the advantion-diffusion and the stokes problem. SIAM Journal on Numerical Analysis, 41(3):1131-1162, 2003. MR2005198(2004g:65157)

[68] J. J. H. Miller, E. O'Riordan, and G. I. Shishkin. Fitted Numerical Methods For Singular Perturbation Problems. World Scientific, 1996. MR1439750 (98c:65002)

[69] D. S. Mitrinovic, J. E. Pecaric, and V. Volenec. Recent Advances in Geometric Inequalities. Mathematics and its applications: East European Series 28, 1989. MR1022443 (91k:52014)

[70] E. Nadler. Piecewise linear best $L_{2}$ approximation on triangulations. In C. K. Chui, L. L. Schumaker, and J. D. Ward, editors, Approximation Theory, volume V, pages 499-502. Academic Press, 1986. MR0903679 (88e:41003)

[71] M. Picasso. An anisotropic error indicator based on zienkiewicz-zhu error estimator:application to elliptic and parabolic problems. SIAM J. SCI. COMPUT., 24(4):13281355, 2003. MR1976219 (2004e:65124)

[72] M. Picasso. Numerical study of the effectivity index for an anisotropic error indicator based on zienkiewicz-zhu error estimator. COMMUNICATION IN NUMERICAL METHODS IN ENGINEERING, 19:13-23, 2003. MR1952014 (2004c:65131)

[73] H. Pottmann, R. Krasauskas, B. Hamann, K. Joy, and W. Seibold. On piecewise linear approximation of quadratic functions. J. Geometry and Graphics, 4(1):31-53, 2000. MR1789628 (2001i:65022)

[74] W. Ren and X. Wang. An iterative grid redistribution method for singular problems in multiple dimensions. J. Comput. Phys., 159:246-273, 2000. MR.1752617

[75] M. C. Rivara. Design and data structure for fully adaptive, multigrid finite element software. ACM Trans. Math. Soft., 10:242-264, 1984. MR0791990 (86f:65207)

[76] M. C. Rivara. Mesh refinement processes based on the generalized bisection of simplices. SIAM Journal on Numerical Analysis, 21:604-613, 1984. MR0744176 (85i:65159)

[77] H. G. Roos, M. Stynes, and L. Tobiska. Numerical Methods for Singularly Perturbed Differential Equations, volume 24 of Springer series in Computational Mathematics. Springer Verlag, 1996. MR1477665 (99a:65134)

[78] R. Schoen and S. Y. Yau. On univalent harmonic maps between surfaces. Invent. Math., 44:265-278, 1978. MR0478219(57:17706)

[79] E. G. Sewell. Automatic generation of triangulations for piecewise polynomial approximation. In Ph. D. dissertation. Purdue Univ., West Lafayette, Ind., 1972.

[80] R. B. Simpson. Anisotropic mesh transformations and optimal error control. Applied Numerical Mathematics, 14:183-198, 1994. MR1273824

[81] J. H. Smith and A. M. Stuart. Analysis of continuous moving mesh equations. SIAM J. Sci. Statist. Comput, 13:1265-1286, 1992.

[82] J. Xu and Z. M. Zhang. Analysis of recovery type a posteriori error estimators for mildly structured grids. Mathematics of Computation, pages 781-801, 2003. MR2047081 (2005f:65141)

[83] P. A. Zegeling. $r$-refinement for evolutionary PDEs with finite elements or finite differences. Appl. Numer. Math., 26B:97-104, January 1998. MR:1602836

[84] P. A. Zegeling. Moving grid techniques. In B. K. S. F. Thompson and N. P. Weatherill, editors, Handbook of Grid Generation, pages 37-1 - 37-18. CRC Press LLC, 1999. MR1848160 (2002f:65001)

[85] Z. M. Zhang and A. Naga. A new finite element gradient recovery method: Superconvergence property. SIAM J. Sci. Comput., 26(4):1192-1213, 2005. MR2143481 (2006d:65137)

[86] O. C. Zienkiewicz and J. Z. Zhu. The superconvergence patch recovery and a posteriori error estimates. Part 1: The recovery techniques. International Journal for Numerical Methods in Engineering, 33:1331-1364, 1992. MR1161557 (93c:73098) 
[87] O. C. Zienkiewicz and J. Z. Zhu. The superconvergence patch recovery and a posteriori error estimates. Part 2: Error estimates and adaptivity. International Journal for Numerical Methods in Engineering, 33:1365-1382, 1992. MR.1161558 (93c:73099)

Department of Mathematics, Pennsylvania State University, University Park, PennSYLVANIA 16802

E-mail address: chenlong@psu.edu

Department of Mathematics, Pennsylvania State University, University Park, PennSYLVANIA 16802

E-mail address: sun@math.psu.edu

The School of Mathematical Science, Peking University, Beijing, People's Republic of China; and Department of Mathematics, Pennsylvania State University, University Park, Pennsylvania 16802

E-mail address: xu@math.psu.edu 